\title{
Représentations des textes et des savoirs chez Charles Estienne : la « vive parole» d'un humaniste
}

\author{
HÉLÈNE CAZES \\ University of Victoria
}

\begin{abstract}
Homme aux savoirs multiples et homme de vulgarisation, Charles Estienne (1514-1564) s'intéressa à la traduction et à l'édition théâtrale parallèlement à ses activités éditoriales et scientifiques, tant en latin qu'en français. Non pas en marge, mais au centre d'une carrière consacrée à la parole du partage des savoirs, l'intérêt pour le théâtre de Charles Estienne se manifeste d'abord par la traduction française d'une comédie italienne contemporaine, rééditée au moins deux fois, puis par des éditions annotées pour la jeunesse d'une comédie de Térence, l'Andrie, qui se continuent par la traduction française de cette comédie, accompagnée d'un traité sur les Jeux des Anciens. Ces deux pièces de théâtres sont représentatives de l'entreprise de Charles Estienne visant à rendre accessible à un plus grand public les grandes œuvres du passé classique comme de la modernité italienne. Surtout, ces textes sont conçus pour la représentation, dans un cadre éducatif mais aussi, simplement, pour le plaisir du spectacle théâtral. La mise en français, mais aussi la mise en lisibilité (par des lexiques, annotations, commentaires, abrègements etc.) paraissent de fait constituer une mise sur scène du texte source, qui sera dit lors de la représentation mais également par son médiateur, le vulgarisateur (qui traduit, édite, rend compréhensible et diffuse). Ainsi, la traduction pour la scène illustre une parole humaniste, de la transmission et du partage des savoirs : une représentation de la reprise et de la vulgarisation.
\end{abstract}

A man of much learning and a man of popularisation, Charles Estienne (1514-1564) was interested in theatrical translation and edition both in Latin and in French, as well as in many other editorial and scientific activities. Interest in theatre was at the centre of Charles Estienne's career consecrated to knowledge sharing; it manifested itself first in the French translation of a contemporary Italian comedy, re-edited at least twice. Then he produced several annotated editions for young people of a comedy by Terence, Andria. These were followed by the French translation of this same comedy, accompanied by a treatise on the Jeux des Anciens [Plays of the Ancients]. These two plays are representative of Charles Estienne's endeavour to make the great works of from classical past as well as contemporary Italy accessible to a wider public. Above all, these texts are designed for performance, in an educational context but also simply for the pleasure of theatrical spectacle. The rendering of the French, and the rendering of readability (through lexica, annotations, commentaries, abridgements etc.) stage the source text, which would be spoken during the performance but also stage its mediator, the populariser (who translated, edited, made comprehensible and disseminated the text). Thus translation for the stage illustrated a humanist position on the transmission and sharing of knowledge: a performance of revival and popularisation. 
$\mathrm{L}$ es textes de la Renaissance emploient de multiples termes, en latin, en français ou en italien, pour désigner spécifiquement les types, genres, formes ou modèles de traductions : interprétation, glose, explication, paraphrase, adaptation... la liste est trop longue pour être exhaustive ${ }^{1}$. Il ne se trouve pas néanmoins, dans cette abondance de termes critiques, de mot désignant spécifiquement « la traduction pour la scène ». Pourtant, le texte dramatique, dans sa complexité, fournit matière à analyse aux rhétoriciens et poéticiens : incomplet sans que soient prises en compte les conditions de sa production et de sa représentation, il ne saurait être traduit par un mot à mot littéral, qui demeurerait incompréhensible sans l'attribution des paroles à des personnages, l'indication d'un décor, celle des mouvements etc. Ainsi, le paratexte, muet ou bavard, qui entoure le texte de théâtre fait partie du texte à traduire, sans tout à fait y appartenir. Hors de tout cadre explicite ou de norme établie, la traduction pour la scène permet ainsi diverses pratiques et se trouve souvent accompagnée de notes, images, commentaires qui restituent la création sur scène ${ }^{2}$.

Dans les années 1540 en France, alors que les poètes de la Troupe qui deviendra la Pléiade défendent et illustrent la langue française en traduisant auteurs classiques et poètes italiens, la traduction et l'inspiration des comédies classiques fondent un théâtre français qualifié de "nouveau " par ses auteurs : la «nouvelle comédie » apparaît d'abord comme " renouveau » de la «nouvelle comédie » illustrée par Térence au second siècle avant notre ère. Or, la « nouveauté » de ces anciennes comédies pourrait bien tenir, essentiellement, à leur mode de traduction et de transmission. Les traductions de l'Andrienne de Térence, données en France par Charles Estienne entre 1540 et 1552, exposent la scène et les coulisses du travail et de l'ambition de leur production : traduite comme une pièce italienne moderne (de 1531), la comédie classique ouvre et partage la liberté de la parole humaniste.

1. Voir Antoine Berman, "tradition, translation, traduction", Le Cahier (Collège International De Philosophie) 6 (1988) : 21-38.

2. Voir Henry S. Turner, Early Modern Theatricality (Oxford : Oxford University Press, 2013) ; Julie Stone Peters, Theatre of the Book, 1480-1880: Print, Text, and Performance in Europe (Oxford: Oxford University Press, 2000) ; C. Dragomirescu, "Vers une typologie des images du théâtre médiéval ", Médiévales 59 (2010) : 63-76 et, surtout, Andrew J. Turner et Giulia Torello-Hill, Terence between Late Antiquity and the Age of Printing: Illustration, Commentary and Performance (Leiden, Boston : Brill, 2015). 
Homme aux savoirs multiples et homme de vulgarisation - au sens propre du terme, la traduction ou adaptation en langue vernaculaire Charles Estienne (1514-1564) s'intéressa à la traduction et à l'édition théâtrale ${ }^{3}$ parallèlement à ses activités éditoriales et scientifiques, tant en latin qu'en français, ainsi que pour les sujets les plus divers et, surtout, les plus en vogue en son temps. Non pas en marge, mais au centre d'une carrière consacrée à la parole du partage des savoirs (anatomie, langues anciennes, histoire, géographie, mais également art des jardins ou marine), l'intérêt pour le théâtre de Charles Estienne se manifeste d'abord par la traduction française d'une comédie italienne contemporaine, rééditée au moins deux fois : la Comédie du Sacrifice, autrement appelée Les Abusez, composée par l'académie siennoise des Intronati, inspirée par Térence ${ }^{4}$. Dans les mêmes années, justement, Charles Estienne donne des éditions annotées pour la jeunesse d'une comédie de Térence, l'Andrienne, qui sont développées par une traduction française de cette comédie, précédée d'une épître sur l'histoire du théâtre - dont l'essentiel est par ailleurs publié séparément comme Brief Recueil sur les Jeux des Anciens ${ }^{5}$. Ainsi, la production de livrets pour la jeunesse, à laquelle appartiennent les rééditions et traductions de l'Andrienne de Térence, se trouve bientôt continuée par une traduction à l'usage d'un public plus large, débordant le cadre de l'apprentissage des langues et des classiques. Ces deux traductions de pièces de théâtre sont représentatives de l'entreprise de Charles Estienne visant à rendre accessible à un plus grand public les grandes œuvres du passé classique comme de la modernité italienne. Surtout, ces textes sont conçus pour la représentation, dans un cadre éducatif mais aussi, simplement, pour le plaisir

3. Voir H. W. Lawton, « Charles Estienne et le théâtre », Revue du XVI' siècle 14 (1927) : 336-347.

4. Academia degli Intronati, Comedie à la manière des Anciens et de pareille matière, intitulée Les Abusez, (traduction par Charles Estienne) (Paris : Pierre Roffet, 1540 et Paris : Estienne Groulleau, 1549). Voir Charles Estienne, L'Andrie ; La comédie du sacrifice ou Les Abuses, éd Lucia Zilli, in Théâtre Français de la Renaissance, première série, vol. 6, La Comédie à l'époque d’Henri II et de Charles IX, éd. E. Balmas, M. Dassonville, L. Zilli (Paris : Presses Universitaires de France, 1990), p. 1-180; Lucia Zilli, « La commedia 'Gl'Ingannati' e la sua traduzione francese : due comicita a confronto ", Studi di Letteratura Francese (Bibliotheca dell'Archivum Romanicum, Serie I, Storia) 10 (1983) : 31-51 et Florinda Cerreta, "A French Translation of Gl'Ingannati: C. Estienne's Les Abusez », Italica 54.1 (1977) : 12-34.

5. P. Terentii Afri Comici Andria, éd. Charles Estienne (Paris : Simon de Colines et François Estienne, 1541) ; La premiere comedie de Terence, intitulée l'Andrie (Paris : Andry Roffet, 1542); Charles Estienne, Brief recueil de toutes les sortes de jeux, qu'avoient les anciens Grecz et Romains, et comment ils usoient d'iceulx (Paris : Andry Roffet, 1542). 
du spectacle théâtral. La "mise en francais », mais aussi la « mise en lisibilité » (par des lexiques, annotations, commentaires, abrègements etc.), paraissent de fait constituer une " mise en scène " du texte source, qui sera dit lors de la représentation mais également par son médiateur, le vulgarisateur (qui traduit, édite, rend compréhensible et diffuse).

De fait, l'entreprise théâtrale de Charles Estienne se comprend dans la continuité, sur près de vingt ans (entre 1536 et 1556, à peu près), d'une réflexion sur la médiation du texte dramatique par l'édition imprimée : au moment où la comédie humaniste se développe en France ${ }^{6}$ - notamment avec la traduction en 1541 des Abusez, comédie composée par l'Académie siennoise des Intronati - , les essais de Charles Estienne constituent une exploration du langage dramatique et de sa transcription textuelle. En effet, le texte théâtral n'est qu'un élément du langage de l'œuvre ${ }^{7}$ : la transcription des dialogues n'est pas un substitut à la représentation, qui est l'œuvre en soi, mais la trace, incomplète, d'une unité que le metteur en scène, la troupe, le public mettent ensemble au jour. L'édition scolaire du texte latin de l'Andrienne donne alors à voir les coulisses de la traduction française. Ainsi, avec la publication d'une édition latine annotée et nourrie de gloses linguistiques, de résumés de l'action, d'indications de jeux, d'introductions sur les genres de la comédie, de traités sur l'architecture du lieu théâtral, puis d'une traduction en français, Charles Estienne élabore non pas une traduction mot à mot du texte dramatique mais la translation d'un univers dramatique. Cette démarche s'inscrit dans le projet général d'une fondation culturelle humaniste : un renouveau littéraire et scientifique par le retour aux textes classiques et par leur interprétation. Entre tradition et création, l'auteur invente alors le théâtre du savoir, qui se donne en représentation dans l'Andrienne et dans ses autres publications.

Le projet fondateur de la comédie nouvelle ne saurait donc être séparé de l'œuvre de Charles Estienne : la traduction théâtrale, pour celui en qui les historiens aiment à reconnaître un " précurseur $»^{8}$ de la comédie humaniste en France, est le modèle de sa prise de parole comme éditeur, traducteur et savant ;

6. M.-M. Fragonard, "La Renaissance ou l'apparition du théâtre à texte ", in Le Théâtre en France des origines à nos jours, dir. A. Viala (Paris : Presses Universitaires de France, 1997), 101-154.

7. Voir Pierre Larthomas, Le Langage dramatique (Paris : Colin, 1972), dont j'utilise les catégories d'analyse poétique.

8. Voir, par exemple, Harold Walter Lawton, Térence en France au XVI siècle : Imitation et influence (Genève : Slatkine, 1972, réédition de Paris : Jouve, 1926), p. 336 et 342. 
elle est l'invention d'une " prise de scène ", qui met sur les tréteaux la notion chère aux humanistes du dialogue avec les textes.

Ainsi, la série consacrée à l'Andrienne constitue à la fois un projet et une pratique pour définir la représentation. Entre filiation et interprétation, elle permet l'invention de l'auteur et construit le modèle d'un théâtre des savoirs.

\section{Vulgariser : traduire, éditer ${ }^{9}$}

Les premières publications de Charles Estienne, - fils, frère, oncle et petitfils d'éditeurs-imprimeurs avant de prendre lui-même en charge les presses parisiennes Estienne en 1549 - ont lieu dès 1535 à Lyon et à Paris, tandis qu'il étudie le droit puis la médecine à Padoue avant de devenir le précepteur de Jean-Antoine de Baiff à Venise puis Haguenau : il fait paraître à l'usage des écoliers, des livrets de vulgarisation inspirés pour la plupart des traités de Lazare de Baif, son employeur et protecteur. Entre 1536 et 1545, il donne ainsi le De Re Vestiaria ${ }^{10}$, le De vasculis ${ }^{11}$, le De Re Navali ${ }^{12}$, le De Re Hortensi ${ }^{13}$, le De coloribus, Vinetum ${ }^{14}$, Arbustum ${ }^{15}$, Sylva ${ }^{16}$, Seminarium ${ }^{17}$, Pratum ${ }^{18}$ puis, en 1547,

9. Voir Hélène Cazes, «Le style simple d'une écriture de l'ombre : la poétique de Charles Estienne », in Lire, choisir, écrire : la vulgarisation des savoirs du Moyen Âge à la Renaissance, éd. V. Giacomotto-Charra et C. Silvi (Paris : École des Chartes, 2014), p. 109-131.

10. De Re vestiaria libellus, ex Bayfio excerptus: addita uulgaris linguae interpretatione, in adolecentulorum gratiam atque utilitatem (Paris : Robert Estienne, 1541 [1 ère éd. Lyon, 1536]).

11. De Vasculis libellus, adulescentulorum causa ex Bayfio decerptus, audita uulgari latinarum nocum interpretatione (Paris : Robert Estienne, 1547 [1 1 ère éd. Lyon, 1535]).

12. De Re Navali (Paris : François Estienne, 1537).

13. De Re hortensi libellus, uulgaria herbarum, florum, ac fruticum, qui in hortis conseri solent, nomina Latinis uocibus efferre decens ex probatis authoribus : in adolescentulorum gratiam (Lyon : héritiers de Simon Vincentius, 1536 ; Paris : Nicolas Buffet [pour Robert Estienne], 1536 [rééd. 1539, 1545...]) ; Troyes : sn, 1542).

14. Vinetum (Paris : Robert Estienne, 1537).

15. Arbustum, Fonticulus, Spinetum (Paris : François Estienne, 1538).

16. Sylua, Frutetum, Collis (Paris : Robert Estienne, 1538).

17. Seminarium et plantarium (Paris : Robert Estienne, 1540).

18. Pratum, Lacus, Arundinetum (Paris : Simon de Colines et François Estienne, 1543). 
le lexique bilingue De Nominibus Arborum ${ }^{19}$. Initialement conçus comme des vocabulaires commentés destinés à l'apprentissage tant du latin que des realia désignées par les termes présentés, ces petits ouvrages rencontrent rapidement un succès qui dépasse le public des précepteurs, de leurs élèves et des collèges : ils s'adressent progressivement à un nouveau public, celui de la nouvelle classe cultivée qui cherche dans les modèles anciens une inspiration pour leur propre temps ${ }^{20}$. Ils deviennent, en 1554, le Praedium Rusticum ${ }^{21}$, traduit en 1564 comme Trésor de la Maison Rustique ${ }^{22}$, que Jean Liébault continuera et augmentera avec grand succès. Parallèlement, Charles Estienne publie lexiques ${ }^{23}$, grammaires ${ }^{24}$, et traités de prononciation du latin ${ }^{25}$. C'est dans ce cadre qu'il se consacre à deux traductions de l'Andrienne de Térence, à la compilation, en latin, d'un traité sur les jeux et à la traduction, au moins deux fois publiée, des Abusez. Ces quatre publications eurent une influence avérée ${ }^{26}$ sur les auteurs dramatiques français (entre autres, La Taille, Grévin, Molière...) et on contribué, de fait, à promouvoir une seule vision de la comédie : celle de Térence.

Ce sont les publics qui semblent d'abord distinguer la nature de la traduction et la conception du théâtre entre les éditions de l'Andrienne et des Abusez. Le titre de chaque œuvre, qui indique explicitement le lectorat attendu, oppose ainsi les jeunes gens au public général des « Jeux de Karesme prenant »:

19. De latinis et graecis nominibus arborum, fruticum, herbarum, piscium, et auium Liber (...) cum Gallica eorum nominum appellatione (Paris : Robert Estienne, 1547 [3] ; Paris : Charles Estienne, 1552). 20. Voir Hélène Cazes, "Translation as Editorial Mediation : Charles Estienne's Experiments with Knowledge Dissemination », Renaissance Studies 29.1 (2014) : 36-54.

21. Praedium rusticum (Paris : Robert [II] Estienne, 1554). Voir sur le procédé de compilation de ces livrets, Hélène Cazes, "Jardins, vergers et maisons-bibliothèques : le grand enfermement du livre imprimé, du Praedium Rusticum de Charles Estienne à la Maison Rustique de Jean Liébault ", Voix plurielles. "La maison et le livre ", dir. Bertrand Bourgeois et Élise Lepage, 5.1 (2008) : http ://brock. scholarsportal.info/journals/voixplurielles/article/view/470/444.

22. Trésor de la Maison Rustique (Paris : Jacques du Puis, 1564).

23. Principia elementaria juuenibus maxime accommodata (Paris : Regnaud Chaudière, 1546) ; Les principes et premiers éléments de la langue latine (Paris : Regnaud Chaudière, 1546).

24. Latinae linguae cum graeca collatio ex Prisciano (Paris : Charles Estienne, 1554).

25. De recta latini sermonis pronunciatione et scriptura libellus (Paris : François Estienne, 1541).

26. Voir M.-M. Fragonard, « La Renaissance ou l'apparition du théâtre à texte », p. 101-154. 
L'Andrie, de Térence, rendue par toutes sortes d'explications plus aisée à lire pour l'agréement des jeunes gens Avec le but qu'après cette comédie, les jeunes gens studieux des belles lettres puissent sans peine aborder toutes les comédies du même auteur. On a ajouté une analyse de la structure de la pièce, en français comme en latin, ainsi que des commentaires exposant le sens de certains mots et donnant des expressions en bon latin ; après une comparaison avec la langue de Cicéron, et des bons auteurs, les façons de parler corrompues et dégénérées y sont amendées. [Ma traduction, ainsi que les suivantes. $]^{27}$

Dans cette première version, l'annotation de la comédie de Térence comprend une traduction partielle mot à mot, en français, avec des explications grammaticales et lexicographiques. Elle vise à faire comprendre le sens du texte, qui sera appris par cour et représenté par les élèves. Ainsi, ces derniers acquerront vocabulaire et grammaire. Les formes non classiques sont donc corrigées et le texte principal reste et demeure l'original latin.

$\mathrm{Au}$ rebours, la traduction des Abusez, deux fois publiée au moins, ne mentionne ni ne donne le texte italien : pas de pédagogie, pas de philologie, pas de commentaire, mais une pièce en français qui se donne à lire sans son modèle.

Gli Ingannati. Comédie du sacrifice des Professeurs de l'Académie vulgaire Senoise, nommez Intronati, célébrée ès jeux d'un Karesme prenant, à Senes, traduicte de langue Tuscane par Charles Estienne. ${ }^{28}$

Les abusez comédie faite à la mode des anciens comiques premièrement composée en langue toscane par les professeurs de l'Académie siennoise et nominée Intronati ; depuis trad. en françoys par Charles Estienne. ${ }^{29}$

27. P. Terentii Afri Comici, Andria : omni interpretationis genere, in adolescentulorum gratiam facilior effecta. Ut ex hac comoedia, omnes deinde alias ab eodem Comico conscriptas, nullo negotio adsequantur iuuenes bonarum litterarum studiosi. / Addita est constructionis ratio, tum uulgaris, tum etiam Latina: Item scholia, quae selectiorum uocabulorum uim, et bene latinarum locutionum formulas contineant : cum Ciceronis, et bonorum authorum sermone conferant : corruptam ac uitiatam loquendi consuetudinem emendent (Paris : Simon de Colines and François Estienne, 1541 ; rééditions Paris : Robert Estienne, 1546 et 1548 ; Paris : François Estienne, 1547 ; Paris : Estienne Groulleau, 1552 et Lyon : Thibaud Payan, 1547 et 1555).

28. Paris : Pierre Roffet, 1540, page titre.

29. Paris : Estienne Groulleau, 1549, page titre. 
Ce contraste entre langues, titres et formats ne doit pas masquer la profonde unité entre les deux démarches : édition annotée et traduction. Néanmoins, si la première fait comprendre, la seconde fait voir : elle donne le plaisir de la représentation. La préface de la traduction des Abusez est ainsi adressée au Dauphin, pas un élève certes, mais tout de même un jeune homme. Elle insiste à trois reprises sur le plaisir du spectacle et du texte, mais elle encadre également l'expérience théâtrale dans une verticalité historique et philologique. Ainsi, elle célèbre l'omniprésence des Anciens, même en première phrase de l'épître sur une comédie créée en 1531 à Sienne. Elle déplore, en contrepoint, la pauvreté des modernes, leur « rudesse » et, en un développement qui annonce la Défense et Illustration de Du Bellay, construit l'éloge du français par un paradoxal regret touchant des œuvres «vulgaires ». Un court exposé sur la nouvelle comédie chez Térence et sur le plaisir du spectateur donne lieu à la présentation des lieux et architectures du théâtre antique avant de conclure sur la noblesse des langues anciennes et, possiblement, modernes.

\section{Epitre du traducteur au Dauphin de France ${ }^{30}$}

Je ne puis assez louer, Monseigneur, la coutume des Anciens, en leurs comédies qu'ils appelaient nouvelles et la façon de disposer et poursuivre leur sens et argument en icelles, pour donner récréation aux auditeurs. Laquelle manière, si jusque à nous ce jourd'hui était parvenue, je ne crois point que ne fussions aussi heureux en ce cas qu'ils étaient, considéré que notre langage, tant pour exprimer, comme aussi pour orner et décorer quelque chose, n'est de rien pour le présent inférieur au leur, combien que pour la plupart du leur soit descendu.

[...] cette présente comédie, ja soit que des Anciens n’ait été faite, mais de bons et modernes esprits sénois, studieux de toute antiquité et honnêteté, faisant de leur langage tuscan une profession et académie, qu'ils nomment Intronati ; toutefois, en lisant, j'espère que la trouverez telle que, si Térence même l'eût composée en italien, à peine mieux l'eût-il su diter, inventer ou déduire.

[...] Bien est vrai que la plupart des Italiens que j'ai nommés, et semblablement tous nos Français, se sont contraints aux rimes de leur

30. Épître dédicatoire aux Abusez (Paris : Roffet, 1540), fol. Aii, Aiiijvo ${ }^{\circ}$ [Aiiiiiii], Éd.Sabine Lardon sur le site Les Idées du théâtre http ://www.idt.paris-sorbonne.fr/html/Estienne-Abuses-Preface.html (site consulté le 18 août 2015). 
langue, comme aussi les Anciens ont toujours fait à leurs mètres ; mais les bons personnages compositeurs de cette comédie, voyant que les vers ôtent la liberté du langage et propriété d'aucunes phrases ont beaucoup mieux aimé faire réciter leur comédie en belle prose (pour mieux montrer l'effet et sens d'icelle) que de s'assujettir à la rime.

À cette cause, je la vous ai bien voulu traduire en notre vulgaire français $[\ldots]$

Doublement nouvelle - puisqu'elle est la nouvelle expression de l'ancienne nouvelle comédie et porte la nouveauté de son apparition chez les Anciens dans sa Renaissance chez les humanistes -, l'œuvre italienne est présentée comme une œuvre ancienne, comme, si de fait, elle venait de Térence : la réserve rhétorique " ja que des Anciens ne soit faite » souligne, en effet, non pas la différence, mais la ressemblance avec les modèles antiques. Second par rapport à l'invention, libre quand il est en prose, le langage est évoqué dans le tout dernier paragraphe comme une modalité, non essentielle, de l'œuvre. Ce texte dernier est seul retenu, pour sa capacité de donner à la fois plaisir et raffinement; mais il prend sa valeur par la profondeur de sa tradition antique et par l'élévation stylistique.

Les mêmes thèmes et termes, voire le même argumentaire, figurent, en 1542, dans la préface de l'Andrie ${ }^{31}$, traduction française de l'Andrienne par Charles Estienne, publiée chez le même éditeur, Roffet, qui joint à la nouveauté de la comédie en prose française, la tradition classique antique. L'éloge de Térence y fait place à l'histoire de la comédie, puis des lieux du théâtre et, enfin de la prose. Commençant par l'ancienneté du modèle, et non plus la double nouveauté de l'original traduit, l'épître qui sert d'introduction insiste sur le théâtre présent et prétend lui fournir un modèle : « montrer le style, la manière de procéder et le bon esprit ».

Pour autant que plusieurs me semblent beaucoup travaillez à entendre la rayson \& maniere des Comedies anciennes, à sçavoir comment elles se faisoient, \& le lieu où elles se iouoyent, \& par quel moyen on en usoit : à ceste cause ie vous ay bien en bref icy voulu déclarer ce que i'en ay pu comprendre par les bons auteurs, \& aussi par les vestiges qui restent

31. Charles Estienne, La premiere comedie de Terence, intitulée l'Andrie (Paris : Antoine Roffet, 1542), préface, cahier signé Aiij. 
auiourd'huy des choses antiques, tant au païs d'Italie, comme en Provence, \& aultres lieux de la noble France, Et l'ay fait principalement, à fin que l'on prenne desormais quelque goust à l'autheur, qui entre les anciens a esté tousiours estimé le bien eloquent, \& tres excellent compositeur de Comedies. Faulte duquel entendre\& la maniere qu'il avoit/ vº̀̀ bien disposer et ordonner le sens et la matière dont il écrivait, notre vulgaire du jourd`hui est tumbé en telle ignorance et cécité que ce qu>il fait pour le présent en telles matières ne sent rien moins que sa comédie. Parquoy m>est aussi venu en fantaisie de traduire et mettre en prose en notre langue vulgaire la première des comédies dudit Terence, seulement en prose, pour plus facilement montrer le stile, la manière de procéder et le bon esprit qu'avait le Comique en la façon d'icelles. ${ }^{32}$

Les deux traductions françaises semblent donc répondre au même souci de fondation d'une littérature nouvelle, nationale, en langue vulgaire que le modèle ancien anoblirait. Le retour à l'antique est présenté comme une reconnaissance de soi et l'imitation comme identité :

Rien ou bien peu de chose avons maintenant en usaige (Lecteur) que nous n'ayons prins des anciens, ou bien que à l'imitation d'iceulx nous n'ayons observé \& ensuyvi au plus pres que faire nous a esté possible : considéré mesmes que la meilleure partie de nostre langaige depend des anciens propos \& maniere de dire. ${ }^{33}$

Si « la meilleure partie » des Modernes est ancienne, jusque dans leur langue, le déclin est néanmoins le sujet principal de la comparaison élaborée entre comédies antiques et comédies contemporaines, dans l'une comme dans l'autre des traductions. Ce n'est pas leur nouveauté en 1540 ou 1542 mais, justement, leur qualité ancienne qui fait la valeur des Abusez, comme de l'Andrienne, et qui leur confère le statut d'exceptions fondatrices : ces pièces représentent l'esprit de Térence en français, par l'imitation du modèle, pour la première, ou par sa traduction, pour la seconde.

32. Charles Estienne, L'Andrie, 1542, aii $\mathrm{v}^{\circ}$.

33. Charles Estienne, Brief recueil de toutes les sortes de jeux, qu'avoient les anciens Grecz et Romains, et comment ils usoient d'iceulx (Paris : Andry Roffet, 1542), p. Aii et Aii vo. 


\title{
Les coulisses de la traduction : l'ABC de la comédie nouvelle
}

Entre la traduction de l'italien, en 1540, et la traduction du latin, en 1542, le petit volume de 1541, destiné à la jeunesse, fournit une édition annotée de l'Andrienne, où Charles Estienne, pour parler comme lui, " démontre » l'art de la traduction théâtrale. Dès 1540, il reprochait aux auteurs français « descendus » des auteurs antiques un défaut de composition que l'imitation ne savait prévenir ni réparer : ils n’avaient pas « encore observé la manière de taire et supplier ce que facilement sans exprimer se pourrait entendre ». Dans l'épître dédicatoire des Abusez, il opposait ainsi la « syncope » moderne et la clarté ancienne :

\begin{abstract}
Mais à ce que maintenant j'en aperçois, notre rude vulgaire a fort syncopé la manière ancienne en matière de comédie, ou pour autant qu'elle lui semblait de trop de frais, ou, possible, pour ce qu'il ne l'entendait point bonnement, de sorte que pour notre comédie vulgaire n'avons retenu qu'un acte simple de leur comédie nouvelle, n'ayant encore observé la manière de taire et supplier ce que facilement sans exprimer se pourrait entendre, qui est un des points en quoi les anciens facteurs mettaient plus de peine, dont est advenu qu'en plusieurs de nos comédies ne se trouve sens, rime ni raison, seulement des paroles ridicules, avec quelque badinage, sans autre invention $\mathrm{n}[\mathrm{i}]$ conclusion. ${ }^{34}$
\end{abstract}

La « syncope ${ }^{35}$ désigne, bien sûr, le raccourcissement de la comédie dans sa forme, qui passerait de trois ou cinq actes à un seul acte (comme dans la farce), de la forme. Mais le terme rappelle également le latin syncopa et le grec б৩үколи́: il connoterait alors une diminution bien plus essentielle, qui brise, ou détruit de fond en comble par coups répétés, coupure, ou ablation. De fait, c'est ce qui est donné à comprendre sans être dit qui, à répétition, manque à la comédie que Charles Estienne appelle "vulgaire », c'est-à-dire l'art de dire sans dire. Ce qui n'est pas «supplié » (au sens de suppléer, produire, fournir), ce ne sont pas des

34. Épître dédicatoire aux Abusez (Paris : Roffet, 1540). Éd.Sabine Lardon sur le site Les Idées du théâtre. 35. Dictionnaire de l'Académie Française (Paris : Veuve Coignard, 1694), p. 521 : «SYNCOPE. s. f. Figure de grammaire, qui consiste dans le retranchement d'une lettre ou d'une syllabe au milieu d'un mot. Dans le vieux langage quand on disoit, je donray, pour je donneray, je lairray, pour, je laisseray, c'estoit une syncope. ingenument pour ingenuëment est une syncope. » 
mots ni mêmes des indications : c'est la manière de composer un univers sans le décrire de manière exhaustive. Du coup, les comédies modernes - mais non nouvelles - semblent incompréhensibles dans leur manque de cohérence.

Nous voici au cœur de la question dramatique : comment dire ce qui n'est pas dans le texte, et, d'abord, comment le lire ? L'Epistre du translateur au lecteur... pour l'Andrie, en 1542, insiste sur la construction du sens par le traducteur et sur ce qui est suppléé, au-delà de la traduction du texte. Le choix de la prose est celui du paraphraste, qui restitue non les vers mais leur sens :

[...] je vous ai bien en bref ici voulu déclarer ce que j'en ai pu comprendre par les bons auteurs, et aussi par les vestiges qui restent aujourd'hui des choses antiques...

Parquoy m'est venu aussi en fantaisie de traduire et mettre en nostre langue vulgaire la première des comédies dudit Térence, seulement en prose, pour plus facilement montrer le stile, la manière de procéder et le bon esprit qu'avait le comique en la façon d'icelles. Je ne l'ai point mise en rithme pour deux causes : l'une pour autant que la liberté d'un traducteur, tels que les Grecs appelaient Paraphraste (c'est-à-dire qui rend les sens, la phrase et l'esprit d'une matière sans contrainte de langage), facilement se perd sous la sujetion du vers; l'autre, à cause que vous avez en ce royaume grandement de bons rithmeurs, lesquels facilement après cette première traduction la pourront mettre en meilleure rithme que je ne saurais faire. Il me suffit d'avoir rendu le principal sens $[\ldots]^{36}$

La première et nécessaire condition de la compréhension du texte de Térence est la connaissance de la langue, qui est fournie au lecteur par l'explication lexicale et grammaticale du texte dans l'édition annotée de 1541. Interprétation du texte, dans la fragmentation du mot à mot et de l'appareil para-textuel des constructions syntaxiques et des scholies, l'édition de l'Andria à usage de la jeunesse mène pas à pas à la construction du sens. Mais le texte théâtral demande, au-delà de son interprétation philologique, sa mise en situation, qui donnera non seulement les didascalies attribuant les répliques à l'un ou l'autre des personnages, mais encore les conditions de sa représentation. La liste des personnages, leur costume, leur apparence générale, le décor font partie de ce sens à «faire passer » et que le 
traducteur recueille de la tradition comme de l'examen attentif du texte. De fait, cette interprétation éclaire les coulisses de la traduction.

Ainsi, l'entrée en scène des personnages de l'Andrienne est déjà une énigme pour qui n’a pas écouté le prologue et lu l'argument de la pièce : Simon veut marier son fils Pamphile avec Philumène, la fille de Chrémès ; Pamphile veut aussi se marier, mais avec Glycère. Chrémès aide le jeune homme à faire échouer le mariage. Sosie, le serviteur de Simon, aide Pamphile en disant qu'il aide Simon, et, de ce temps, Glycère enfante dans la douleur et donne naissance à un fils qui doit être reconnu. Pour commencer l'écheveau des manipulations, mensonges et secrets, la première scène fait entendre les confidences de Simon à son affranchi Sosie : il prépare un repas de noces non pas pour célébrer un mariage mais pour obliger son fils à déclarer son refus du mariage... Pièce de jeunesse de Térence, qui l'aurait composée à l'âge de 18 ans, la comédie est proposée à la sagacité des jeunes apprentis-latinistes, qui ont besoin de savoir qui est qui et qui dit quoi, mais aussi qui ment à qui, qui se cache de qui, etc.

Fondée sur la connaissance de la langue comme de la tradition savante, l'élucidation de la comédie de Térence par Charles Estienne permettrait, du moins en théorie, de la faire jouer, en latin, par les élèves. Une telle pratique est attestée dans de nombreux collèges, en liaison avec la pédagogie humaniste des colloques et dialogues ${ }^{37}$, chez les précepteurs de la famille Estienne par un souvenir d'enfance du neveu de Charles, Henri (II) Estienne (1531-1598), à qui était dédié en 1536 le petit recueil De Re Hortensi. En 1564, dans sa préface aux Poètes épiques grecs, il raconte comment il tomba amoureux de la langue grecque en écoutant une représentation de la Médée d’Euripide :

Je me souviens que les premières étincelles de cet amour (si je peux m'exprimer ainsi) furent allumées en moi lorsque, tout jeune encore, je fus confié à un précepteur qui interprétait avec ses autres élèves la Médée d'Euripide. Chaque fois que j'assistais à cette pièce (et, par-dessus tous les autres exercices, mon précepteur aimait fort à donner ce genre

37. Voir Eva Kushner, Le dialogue à la Renaissance : histoire et poétique (Genève : Droz, 2004); Bernard Colombat, La grammaire latine en France à la Renaissance et à l'Âge classique : théories et pédagogie (Grenoble : Ellug, 1999) ; Jacques Chomarat, "Paideia et Rhétorique chez Érasme », Bulletin de l’Association Guillaume Budé 1.1 (1986) : 67-78, et Mathieu Ferrand, « Le théâtre de Barthélemy Aneau. Écriture dramatique et pédagogie de l'actio ", Cahiers de recherches médiévales et humanistes 22 (2011): 363-378. 
d'entraînement aux enfants), la mélodie des mots du grec, si douce, si voluptueuse, s'emparait de moi et me charmait; je n'y entendais d'ailleurs rien d'autre que des sons! De ce moment, je fus hanté par cette unique pensée, tout mon esprit ne tendit plus que vers cet unique but : que, de spectateur, je devienne moi aussi, enfin, acteur ${ }^{38}$.

La représentation chez le précepteur du jeune Henri indique certainement l'intention de l'interprétation de 1541. L'intérêt de Charles Estienne et ses publications sur la prononciation du latin corroborent ce projet de mise en scène scolaire, privée, du texte antique. En effet, en cette même année de 1541, il fait paraître un court traité au long titre sur les bons usages de prononciation, présenté comme une méthode d'apprentissage de la langue, le De recta latini sermonis pronunciatione et scriptura libellus ${ }^{39}$. Ce livret, paru chez son frère François Estienne, s'intègre à la vision d'une pédagogie du « latin vivant » ainsi que l'illustre son sous-titre : "ob hoc maxime aeditus ut nostri adolescentuli facilius condiscant, eam linguam, cui quotidie dant operam, apte, distincte, ornate, pronunciare et scribere " [composé avec le but premier que nos jeunes gens puissent facilement a prendre cette langue en l'utilisant chaque jour pour prononcer et écrire avec exactitude, clarté et élégance. $\left.{ }^{40}\right]$

La préface du manuel présente la " mise en parole » comme la meilleure stratégie éducative pour l'apprentissage de la langue et se continue par une conversation avec un détracteur fictif :

- Mais, me diras-tu, ces préceptes de prononciation me semblent très difficiles; et ceux qui sont facilement appris changent sans arrêt !

38. Henri Estienne, Poetce Groci principes heroici carminis (Genève : Henri Estienne, 1564), *.ii : "Primus autem huiusce mei amoris igniculos (ut ita loquar) inde accendi memini, quod quum in prima pueritia praeceptori cuidam traditus essem qui Euripidis Medeam aliis suis discipulis interpretabatur, quoties eos fabulam hanc agentes spectabam, (summe enim illi meo praeceptori haec pueros exercendi ratio placebat) tanta dulcedine voluptateque meas aures illa Graecarum vocum modulatio titillabat (quas alioqui non ultra sonum intelligebam) ut ab eo tempore unum hoc noctes diesque versarem, et in hoc uno contendendos omnes ingenii neruos existimarem, ut fabulae, cuius spectator saepe fuissem, actor et ipse tandem euaderem. »

39. Charles Estienne, De recta latini sermonis pronunciatione et scriptura libellus (Paris : François Estienne, 1541).

40. Charles Estienne, De recta latini sermonis pronunciatione et scriptura libellus (Paris : François Estienne, 1541). Ma traduction. 
- Ils sont en vérité bien plus faciles qu'on ne le croirait, surtout ceux que tu déduiras de ce qui va suivre et qui te semblent évidents. [...]

Il me faut donc veiller à ce que, grâce à notre petit livre, nos chers jeunes gens acquièrent des principes sur la prononciation et sur l'écriture qui soient pour ainsi dire légers et bien plus utiles. Qu'ensuite pour de pareilles questions, ils ne soient plus insultés, ou accusés d'être des barbares de naissance ! $[\ldots]^{41}$

En 1555, c'est encore sous forme de dialogue qu'il propose les Rudimenta Latino Gallica, cum accentibus ${ }^{42}$, un cours de grammaire et de vocabulaire à l'usage des écoles et des précepteurs. Là aussi, il insiste sur l'articulation des mots, et leur accent, définissant la voix comme le mot prononcé. Or, cette prononciation fait partie du sens non écrit : en opposant " voix articulée ", " voix confuse » et "voix modulée », Charles Estienne revient à ce qui doit être observé même si rien dans les lettres ni les mots du texte ne l'indique :

Toute voix Articulee, ou confuse. Articulee, signifie autant comme distincte. Voix articulee, est celle qui est expliquee \& distinctement proferee par la parolle des hommes. Et est autrement appelee literale, pour ce qu'elle peult estre escripte par lettres. Voic confuse, est celle qui est faicte par un simple son des bestes, \& animaulx irraisonnables, \& ne peult estre escripte: comme le hennissement d'un cheval, le meuglement ou buglement d'un taureau, le cuignement dun porc, le beellement d'une brebis ou mouton. Aussi est adiousté la voix modulee, c'est a dire, faicte par modulation \& chant mesuré de fleutes, orgues, \& autres instruments musicaulx. Laquelle ia soit que ne se puisse escrire, si ha elle aussi une distinction. ${ }^{43}$

41. De recta latini sermonis pronunciatione, 2 : «At uero (inquies) difficillima uidentur pronunciationis praecepta : et ista inconstantia, quae ab omnibus facile peruentantur. Imo certe faciliora sunt quam quis credat ; praesertim ea quae latius patere ex sequentibus iudicabis. (...) Danda igitur est opera ut hoc libello nostri adolescentuli praecepta quaedam leuia et magis utilia rectae pronunciationis ac scripturae condiscant, quibus posthac non amplius insulsi aut ad barbariem nati iudicentur. »

42. Charles Estienne, Rudimenta Latinogallica, cum accentibus (Paris : Charles Estienne, 1555).

43. Rudimenta Latino Gallica cum accentibus (1555), a iii. 
La parole dépasse ainsi le mot, tout comme la représentation théâtrale déborde la littéralité du texte. En tous les cas, la prononciation, non indiquée dans la transcription de la pièce, est essentielle pour la compréhension de la nature de la comédie.

L'interprétation se comprend alors comme une lecture, linéaire, du texte qui sera appris et joué. Elle comprend, outre le cours de langue, des éléments dramatiques qui dépassent le texte, comme la reconnaissance des « voix confuses » et des actions qui ne seraient pas explicitées. Tenant lieu des illustrations et des gloses qui accompagnaient et éclairaient le texte de Térence durant sa transmission manuscrite et imprimée, le commentaire linéaire de Charles Estienne reprend une tradition scolaire riche et vivace d'élucidations dramatiques de Térence ${ }^{44}$, qui figurait comme commentaire dans les éditions de la Renaissance ${ }^{45}$. Ainsi, l'édition de l'Andria donnée à Sélestat en 1520 par Nicolas Gerbel ${ }^{46}$, comprenait la Vie de Térence tirée du recueil sur les poètes latins de Petrus Crinitus, puis l'épître au lecteur sur le commentaire (qui serait tiré de Priscien) et enfin le commentaire en partie double : interlinéaire pour la langue, et continu, sur une colonne extérieure de texte (à droite sur la page de droite, à gauche sur la page de gauche) pour l'explication de l'action, des mouvements des personnages et du jeu. Des illustrations renseignent le lecteur sur le décor et les costumes. Le livre, somptueux, rappelle les manuscrits glosés des sentences ou de la Bible ... un texte espacé, central, une petite glose interlinéaire et un encadrement par le commentaire suivi. Charles Estienne simplifie et désacralise le modèle dans son livret, sans illustration ni hiérarchie du texte. S'y succèdent, en une longue et inégale colonne de texte pièces liminaire, arguments, extraits de la pièce de théâtre, constructions et scholies. La glose interlinéaire et le commentaire continu ont laissé place à un «théâtre de la lecture ", en procession :

44. Voir Harold Walter Lawton, Térence en France au XVI siècle : Imitation et influence (Genève : Slatkine, 1972. réédition de Paris : Jouve, 1926) et John H. Grant, Studies in the Textual Tradition of Terence (Toronto, Buffalo, Londres : University of Toronto Press, 1986).

45. Voir Ludmilla Evdokimova, « Commentaires des comédies de Térence dans l'édition de Vérard et leurs sources ", Le Moyen Français 54 (2004) : 95-152 ; Axel Christoph Gampp, «Le langage des gestes dans les manuscrits de Térence antérieurs au XIIIe siècle ", Sénéfiance 41 (1998) : 219-236.

46. Pub. Terentii Aphri Poetae Comici Lepidissimi Andria [...] (Selestat: Schurerius, 1520). 
- la préface au lecteur « amousos", sans culture

- les iambes de Volcatius Sedigitus sur les comiques latins (un poème mnémotechnique transmis par Aulu-Gelle ${ }^{47}$ )

- la vie de Térence par Petrus Crinitus

- un index des expressions propres à Térence ${ }^{48}$

- puis, à partir du premier cahier folioté (page 1) et signé (A), les personnages

- l'argument donné par Donat

- Les Periocha de Caius Sulpicius Apollinaris

- Et enfin, précédé de son argument, le texte, scène par scène, toujours suivi de sa construction et des scholies.

- le folio 201 porte la mention « Finis »

- un dernier cahier répertorie les traductions françaises données au cours du livre et fournit la liste alphabétique des " corrections linguistiques » apportées au latin de Térence. ${ }^{49}$

Tandis que les éditions de Sélestat, Lyon ou Paris distinguaient les paratextes de la comédie par une mise en page structurée et hiérarchisée, l'édition de Charles Estienne découpe, fragmente et tisse les niveaux de texte et commentaires, donnant dans la succession de ses pages un discours qui semble la leçon préparatoire d'une mémorisation par l'élève, donnant d'abord le sens général de la scène, puis une traduction française (la construction), et enfin les points de grammaire à retenir (scolies). Bien souvent, ces éclaircissements comportent des indications de jeu et des récapitulations de l'action, mais aussi des remarques sur la dramaturgie. Ainsi, avant la quatrième scène du premier acte, l'argument annonce l'action (le départ de Mysis, esclave de Glycère, qui va chercher la sage-femme) et commente un procédé dramaturgique : le personnage parle seul pour que le public connaisse ses intentions et comprenne l'action qui va suivre.

47. Aulu-Gelle, Noctes Atticce, livre 15 (consultation en ligne sur http://penelope.uchicago.edu/Thayer/L/ Roman/Texts/Gellius/15*.html le 21 novembre 2014).

48. Index Latinarum locutionum, Scholijs superioribus interpretatarum cuius adminiculo, non solum Andriam, sed etiam reliquas comcedias, a Terentio conscriptas, facile intelligent adolescentuli.

49. INDEX Gallicarum dictionum ac locutionum Scholijs huius Comedice interpretatarum. Item etiam corrupti sermonis emendati Alphabeticus Index. 
ARGUMENT. Mysis quitte Glycère pour aller chercher la sage-femme de Lesbos. En sortant, elle dit à voix haute ce qu'elle va faire et, en même temps, ce qu'elle pense. C'est un procédé de la comédie pour que les spectateurs comprennent le sens de la pièce. ${ }^{50}$

Plus tard, l'argument indique que le personnage de Pamphile est bouleversé à l'idée d'être marié à une autre que Glycère et résiste de plus en plus aux projets matrimoniaux de son père ${ }^{51}$. Ces interprétations des mouvements et des sentiments du personnage sont nombreuses dans les arguments : leur but avoué est d'aider la compréhension du public qui, en l'occurrence, n'est pas spectateur de la pièce mais futur acteur. De ce fait, Charles Estienne indique les émotions du personnage, explicite les quiproquos, etc. Il faut dire que l'Andrie est une comédie à secrets, mensonges, rebondissements, reconnaissances, illusions et malentendus : les jeunes élèves avaient certainement grand besoin de ces élucidations !

La construction et les scholies sont bien plus étoffées que ces courts arguments, en tête de la scène. L'ouverture du texte est ainsi longuement glosée après la fin de la scène : tout d'abord avec une traduction française puis avec les expressions à retenir.

\section{ACTE UN SCÈNE UN}

SIMON, le vieillard, et SOSIE, l'affranchi

SIMON : Portez tout ça dedans et sortez ! Toi, Sosie, viens me voir. Un mot.

SOSIE : Tu l'as déjà dit, non ? Qu'on fasse bien ce que tu as commandé. ${ }^{52}$ $[\ldots]$

50. P. Terentii Afri Comici Andria, (1541), fol. 43 : «ACTUS I SCEN IIII : ARG. Mysis a Glycerio egreditur, $v t$ Lesbiam obstetricem accensat. Egrediens loquitur de eo quod est gestura, \& simul docet quid intus sit gestum, qui mos est comicus: vt facilior sit audientibus comodioe sensus. "

51. P. Terentii Afri Comici Andria, (1541), fol. 45 : " ARG. Ostenditur animus Pamphili valde commotus, ob nuptias: atque adeo magis, magisque per Mysidem excitatur, ad resistendum patri, nuptias inducenti. [On voit que Pamphile est profondément inquiet de ce projet de mariage et, du coup, il est de plus en plus sensible aux encouragements de Mysis qui lui conseille de résister à son père, lequel pousse le mariage.]"

52. P. Terentii Afri Comici Andria, (1541), fol. 7: " ACTUS PRIMUS SCENA PRIMA / SIMO senex SOSIA Libertus / SI. VOS isthæc intro auferte: abite. Sosia, / Adesdum: paucis te volo. / SO. Dictum puta: Nempe ut curentur recte hæc. » 


\section{CONSTRUCTION}

(Hola !) Vous (les esclaves) car Simon est représenté en train de parler aux esclaves qui sont devant la maison et il fait son entrée sur scène sans autre présentation. Vous aultres serviteurs APPORTEZ TOUT CELA DEDANS, prenez, portez ces choses (que je vous montre) à la maison, ou là où elles se rangent portez ces choses leans. Maintenant que demande-til qu'on apporte ? Ce n'est pas difficile à comprendre. Il est probable que Simon, qui fait son entrée sur la scène comme s'il revenait du marché, dit à ses esclaves de porter à l'intérieur la nourriture qu'il rapporte et qu'il achetée pour les noces de son fils (ou pour les noces qu'il pense lui faire...) SORTEZ, éloignez-vous, retirez vous, comme si les esclaves restaient à attendre d'autres instructions ou manquaient d'empressement à porter ce que le maître leur a ordonné de ranger. TOI, SOSIE, VIENS hé, viens là. Viença Sosia. JE TE VEUX QUELQUES. Rétablis les mots sous-entendus : te dire quelques mot. C'est-à-dire : je voudrais te parler un peu Ie te vueil diri ung mot. PENSE QUE C'EST DIT. Crois bien que tu l'as déjà dit. pensez desia l'avoir dit. OUI. C'est-à-dire, en effet. cest a scavoir. QUE CES CHOSES (celles que tu as ordonnées) SOIENT EXÉCUTÉES COMME IL FAUT. Qu'elles soient accomplies avec soin, qu'elles soient faites, que ce qu'avez commandé, soit bien faict. ${ }^{53}$ [...]

\section{SCHOLIES}

Les adjectifs pauca et multa, chez les auteurs de comédies mais aussi, parfois, chez d'autres auteurs n'ont pas besoin d'un nom pour faire sens : ils font comprendre et sous-entendent « mots ». Ausculta paucis, Escoute

53. P. Terentii Afri Comici Andria, (1541), fol. 10 : «CONSTRUCTIO (Heus) VOS (serui) nam ad seruos qui sunt foris se loqui fingit Simo, \& ex abrupto scenam ingreditur Vous aultres serviteurs AUFERTE ISTHAEC INTRO abducite, adportate, ea (quæ vobis ostendo ) domum, vel in sua repositoria portez ces choses leans. Quid autem, sit quod auferri iubeat, minime laborandum est. Verisimile est autem, Simonem ex abrupto pulpitum ingredientem, hoc seruis dicere, tanquam e foro veniens, vt quæ secum adferret edulia, ad nuptias filiij facienda (quas tamen fingebat) intro auferrent ABITE discedite, retirez vous, quasi post primum præceptum immorari viderentur, aut paulo negligentius auferre quod herus iusserat. SOSIA ADESDUM. ehodum ad me. Viença Sosia. VOLO TE PAUCIS. (supple, verbis alloqui, per ecllipsim) hoc est, Volo tecum pauca confabulari. Ie te vueil diri ung mot. PUTA DICTUM. Existima iam te dixisse. pensez desia l'avoir dit. NEMPE scilicet, hoc est. cest a scavoir VT HAEC (quæ pracepisti) CURENTUR RECTE. diligenter administrentur, fiant, que ce qu'avez commandé, soit bien faict. " 
ung petit. Nec multis moror Ie ne m'arrestray pas a luy dire beaucoup de paroles. Cicéron, dans le Pro Cluentio, Ne multis, Diogenes emitur. Pour faire court, Diogenes fut achepté. ${ }^{54}$

L'interprétation de l'entrée en scène de Simon et Sosie comporte bien plus que la traduction linéaire. En ce début de jeu, bien plus longuement que pour les scènes suivantes, elle explicite les référents de cette exposition dramatique tout en réduisant les éventuelles ambiguïtés du texte. Ainsi, elle commence par l'ajout de «Holà ! » qui indique que Simon fait venir à lui ceux à qui il s'adresse. Toujours entre parenthèses, Charles Estienne développe tous les pronoms indéfinis et rétablit les mots sous-entendus par l'ellipse syntaxique usuelle. La phrase latine est suivie par une glose, en latin, proposant plusieurs expressions synonymiques, puis par une traduction française. Surtout, à cette occasion, le commentateur met le texte en scène, il situe les personnages : Simon arrive du marché, les esclaves sont devant la maison, puis les esclaves traînent... Pour ces vers d'exposition, il expose l'espace et présente les personnages dans leur position et mouvements.

Plus loin, les scholies se rapportant à ces quatre premiers vers énoncent que le mot verba (mots) est fréquemment sous-entendu après pauca et multa : ce qu'il faut retenir et réutiliser pour la composition en latin, comme le suggère la citation de Cicéron sur laquelle se clôt l'extrait ${ }^{55}$.

Mais combien de traductions et commentaires pour un vers de Térence? L'accumulation joue contre la continuité du texte. L'édition annotée livre une traduction fragmentaire, injouable en français, coupée et entrecoupée d'indications dramaturgiques et philologiques. Elle mène à la représentation du texte latin mais ne se substitue aucunement à l'expérience originale : au contraire, elle constitue une exposition des coulisses, le plus souvent invisibles, du sens dramatique.

54. P. Terentii Afri Comici Andria, (1541), fol. 19 : «SCHOLIA. Paucis \& multis, comicis, \& interdum apud alios scriptores, sine adiecto signifant: sub se etiam comprehendunt ac subaudiunt, verbis. Ausculta paucis, Escoute ung petit. Nec multis moror Ie ne m'arrestray pas a luy dire beaucoup de paroles. Cicero pro Cluentio. Pour faire court, Diogenes fut achepté. »

55. Cicéron, Pro Cluentio 16. La formule est une cheville, fréquemment utilisée en latin et recommandée dans les manuels scolaires, On la retrouve ainsi dans le dictionnaire Gaffiot, dépositaire des recueils scolaires anciens, comme première citation pour le verbe moror : "Nec multis morer, Cic. Verr. 4, 104. ", Félix Gaffiot, Dictionnaire latin-français (Paris : Hachette, 1934), p. 995. 
Le Therence en françois, publié en 1499 et 1500 chez Antoine Vérard à Paris ${ }^{56}$, réédité à Paris par Bossozel et Kerver en 1539, proposait pour la traduction française, une explicitation comparable de la concision latine. Le traducteur y entremêlait une traduction en prose, au plus près du texte original, et une traduction en vers, plus longue et plus libre. La traduction en prose, la première, en une large colonne, suivait le texte latin donné sans retour à la ligne en marge extérieure. Plus loin, à la fin de la scène, venait la traduction en vers. En regard de quelques vers latins, cités en sa marge comme points de référence, le texte français y prenait deux larges colonnes qui, telle une paraphrase, développaient, amplifiaient et expliquaient l'original. Ainsi, pour le même passage, la traduction française donnait :

SYMO.

Vous qui estes ceand dedans ostez cela : allez hors Sosie soyes present: ie vueil ung pou parler a toy.

SOSIE.

Syre prends que ce que tu veulx dire soit dit. Je scay bien ce que c'est. Tu veux que toutes ces choses soient bien faictes et appareillees a droit.

SYMO.

Je te vueil autre chose..$^{57}$

SYMON

Vos istec intro auferte

Allez: ostez ceste viande

Et la portez a la cuisine

Sans revenir se ne vous mande

Ou se ne vous fais aucun signe

Chascun de vous dicy decline

Fors toy Sosia : vien pres moy

Jay ung peu a parler a toy

SOSIA

Dictum puta

Maistre dy ce quil te plaira

56. Voir Lawton, Térence en France..., p. 356-7 et 450.

57. Charles Estienne, Therence en françois (Paris : Vérard, 1499), fol. a vi. 
Affin que voise appareiller

La viande / car il sera

Tantost saison de la manger ${ }^{58}$

C'est dans le texte à dire sur scène que l'éditeur de Vérard indique qu'il s'agit de nourriture (viande), qu'il faut la porter à la cuisine, que les esclaves doivent aller sans attendre, et qu'il y aura bientôt un banquet. Une illustration précède chaque scène, comme le faisait la tradition manuscrite, et dépeint décor, personnages et positions. La traduction française, en ce cas, est dédoublée entre l'interprétation en prose et la paraphrase en vers, tandis que la gravure et le commentaire justifient les choix de traduction et les expliquent. La « syncope » des modernes multiplie les redondances et les intègre au livre. En ce cas, l'espace de représentation est donc le livre, qui, construit comme un monument étagé, se suffirait à lui-même.

Au rebours, l'Andria de Charles Estienne utilise l'espace du livre comme espace préparatoire à la sortie du texte vers la représentation théâtrale. La traduction y figure comme glose linéaire seulement : en son centre, se lit le texte latin dont la masse des commentaires, en leurs strates, sert la compréhension et la future représentation, non sa translation vers le français. Le texte scolaire est ainsi une propédeutique à la parole dramatique et au jeu : un texte destiné au dépassement et à l'effacement une fois lu et compris, une fois que l'original est « mis sur scène. »

\section{Prises de parole : la vive voix}

Avec la traduction française, la " mise sur scène " scolaire devient mise en scène. Il lui faut tout d'abord un espace dramatique en français : c'est chose faite avec la traduction des Abusez de 1540, puis, en 1542, par le Brief recueil de toutes les sortes de jeux, qu'avoient les anciens Grecz et Romains, et comment ils usoient d'iceulx, publié en 1542 chez le même André Roffet et souvent joint à la traduction de l'Andrienne dans les recueils (comme la réédition par Estienne Groulleau à Paris, en 1555). Dans cette dernière addition, se détermine en effet la nature dramatique de la traduction française, qui fonde un nouveau théâtre français alors que «notre vulgaire du jourd'hui est tombé en telle ignorance et cécité que ce qu'il fait pour le présent en telles matières ne sent rien moins que sa 
comédie » ${ }^{59}$. En recourant aux « auteurs » et " vestiges » de l'Antiquité, Charles Estienne propose de reconstruire genres et architectures du théâtre antique pour reconstruire la représentation de la comédie. Aussi, le traité sur les jeux ne fait pas partie des lexiques et répertoires pour la jeunesse par lesquels Charles Estienne s'était fait connaître. Il s'intègre au contraire à l'écriture dramatique du traducteur, qu'il commente hors du contexte scolaire. De fait, le traité sur les jeux construit la scène où se donnera la comédie nouvelle : un espace pour la parole des personnages qui portera le sens que l'interprétation a restitué dans les arguments et la glose linéaire. De cette manière, la description du théâtre antique complète la traduction en prose du texte : tout comme les gravures du Térence français plantaient le décor du dialogue, le théâtre imaginaire transforme la traduction du texte antique en parole.

Du coup, la traduction du "paraphraste » ainsi que se définit Charles Estienne prend place dans le lieu de parole, sans qu'il soit besoin de le créer dans l'espace de la page, comme le faisait le Therence en françois donné par Vérard.

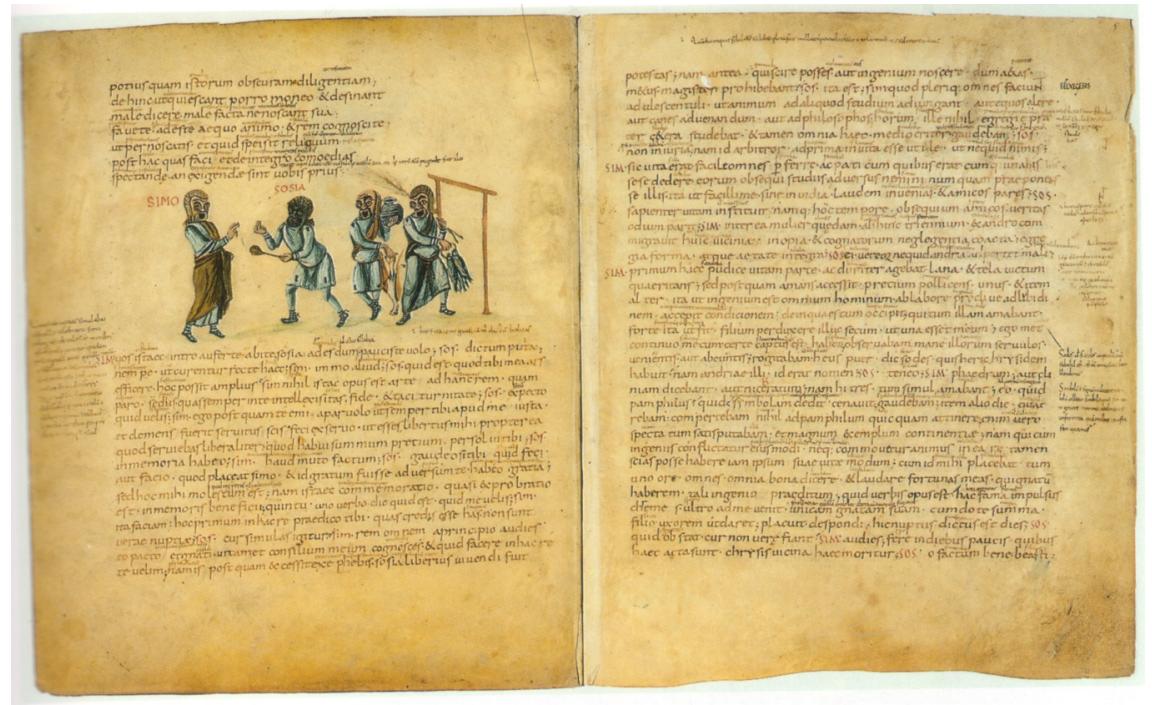

Illustration 1. Vaticanus Latinus 3868, fol. $4 \mathrm{v}^{\circ}-5 \mathrm{r}^{\circ}$. Ce manuscrit enluminé du IX siècle, est la copie faite à Corvey par Hrodgarius, d'un modèle du III siècle. Il comprend 141 illustrations (Charles R. Morey, "The Vatican Terence ", Classical Philology 26.4 
(1931) : 374-385. La tradition représentée par cet exemplaire est abondamment représentée par les témoins manuscrits, jusque dans la disposition du texte (en haut), de sa glose interlinéaire et du commentaire (en prose, après l'image). Cette illustration demeure la source des figurations ultérieures de la première scène. Source : Wikimedia Commons. Domaine Public.

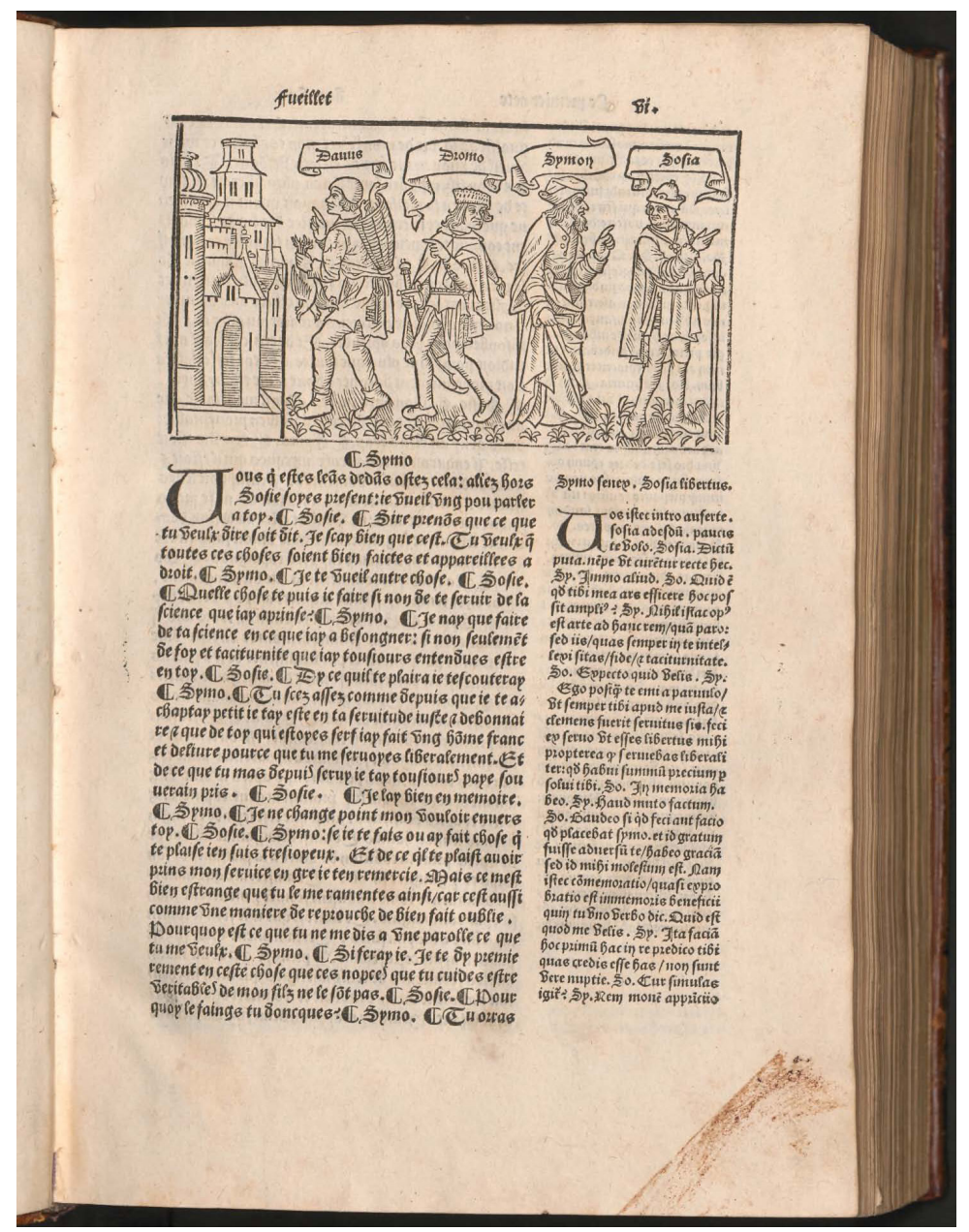

Illustration 2. Therence en françois (Paris : Vérard, 1499), fol. a vi : la traduction en prose dans la colonne intérieure, le texte latin dans la colonne extérieure, comme en marge. La scène s'ouvre dans l'espace créé par la gravure ... les esclaves quittent l'estrade et 
vont ranger les victuailles des noces. Au centre droit, Simon retient Sosie, pris à part. La barbe et le turban de Simon permettent le reconnaître le type du vieillard. Sosie porte le bonnet phrygien des affranchis. Son bâton fait deviner qu'il prendra l'action en charge. Les mains levées et doigts pointés indiquent que seuls ces deux derniers personnages parleront. Le regard vers la droite du second esclave, Dromo, indique qu'il tarde à exécuter l'ordre de Simon. Image publiée sur http://www.archive.org/details/OEXV710 (téléchargement le $1^{\mathrm{er}}$ novembre 2014). Source : Bibliothèque Sainte-Geneviève, OEXV 710 RES. Domaine Public.

Charles Estienne a dressé le tableau de la scène dans le recueil et a réduit les ambiguïtés de l'original en ajoutant des adverbes et des interjections servant d'embrayeurs du discours. Sa traduction se suffit alors à elle-même, indiquant clairement qui est sur scène, qui quitte la scène ( « qu〉on se retire ») en entrant dans la maison ( léans », qui parle à qui ("Sosia »). Il ajoute interjections ( Sa ho ») et apostrophes ( Sire») pour confirmer le statut de Simon comme personnage principal, qui tient la scène et dirige la maison.

SI. Sa ho, portez cela léans, et qu'on se retire, vien ça, Sosia, i’ay à te dire un mot.

SO. Autant que si vous l'aviez desja dit, Sire, nest-ce pas que tout soit bien appareillé ? $^{60}$

Cette traduction nouvelle n'est pas, donc, le simple rapiéçage de l'interprétation linéaire de 1541. Pour les mêmes vers, elle donnait les phrases suivantes :

Vous aultres serviteurs [...] portez ces choses leans. [...] retirez vous, [...] Viença Sosia. [...] Ie te vueil diri ung mot. [...]pensez desia l'avoir dit. $[\ldots]$ cest a scavoir [...] que ce qu'avez commandé, soit bien faict. ${ }^{61}$

La traduction de 1542 est une parole, suivie, donnée en contexte : un texte à " interpréter » sur scène. De fait, cette version française de 1542 s'appuie sur la lecture laborieuse de l'édition latine tout comme sur l'exposé qui met en place l'espace scénique et dramatique. Loin de s'opposer, l'interprétation

60. Charles Estienne, Andrie (Paris : Estienne Groulleau, 1552), p. 30.

61. Charles Estienne, P. Terentii Afri Comici Andria, (1541), fol. 10 : Constructio. 
latine et la traduction française se pensent ainsi comme une démarche unique et cohérente, qui inclut la recherche historique sur les théâtres anciens. Cette rencontre des enjeux se donne à lire dans le terme français "interprétation ", qui désigne en effet l'interpretatio (la lecture commentée par une traduction linéaire expliquée, ou par une paraphrase) mais également, à partir de cet usage, la «traduction effectuée au fur et à mesure qu'un orateur parle $»^{62}$ par opposition à la traduction écrite, différée ; cette évolution sémantique correspond à un autre sens du terme, attesté dès le seizième siècle : l'« action de jouer un rôle $»^{63}$. Ce que révèle la continuité des travaux et traductions de Charles, c'est la dimension cachée de la version finale, en français, créée sur la scène pour et avec un public.

En ce sens, l'Andrienne donnée par Charles Estienne porte, dans la profondeur de ses préparations et dans la résonance avec les autres œuvres et éditions du même auteur, une conception du théatre comme parole en représentation : une parole dont l'éditeur doit donner les contextes (philologiques, dramaturgiques, scéniques, culturels), tout comme le metteur en scène lui donne sens par la création de la pièce. L'appareil interprétatif de l'édition annotée comme du traité sur le théâtre semble alors remédier au défaut du texte : il restitue ce qui manque au texte, ce qui est «muet » dans la transcription des dialogues, mais qui fait partie intégrante de la comédie.

Or, le théâtre revêt chez Charles Estienne, bien au-delà de notre Andrienne, le statut d'un espace où la parole transmet le savoir. Tout comme la représentation met en œuvre, sans les énoncer, les connaissances que consignait l'édition pour la jeunesse - et qui justifient la pratique du théâtre scolaire comme méthode pédagogique - c'est en représentation, dans un théâtre ${ }^{64}$, que Charles Estienne " démontre » l'anatomie du corps humain dans son traité de

62. Trésor Informatisé de la Langue Française, consulté sur le site Analyse et Traitement Informatique de la Langue Française, ATILF, http://atilf.atilf.fr/dendien/scripts/tlfiv5/search. exe?34;s=1253386200;cat=1;m=traduction+simultan\%82e; (le $1^{\mathrm{er}}$ novembre 2016).

63. Trésor Informatisé de la Langue Française http://atilf.atilf.fr/dendien/scripts/tlfiv5/visusel. exe?64;s=1253386200;r=4;nat=;sol=0; (le $1^{\mathrm{er}}$ novembre 2016), sens III A.

64. Voir Hélène Cazes, “ "Démonstrer à l'œil” l'ombre d'une dissection. L'illusion théâtrale du corps humain selon Charles Estienne $(1545,1546)$ ", Cahiers de recherches médiévales et humanistes 29.1 (2015) : 305-349. 
La Dissection des parties du corps humain ${ }^{65}$. Au troisième livre, il décrit ce que devrait être le "théâtre d'anatomie ", en des termes repris du Brief Recueil sur les jeux de 1542, donnant comme modèle pour la transmission et l'élaboration du savoir la scène d'un « spectacle public » :

Tout ainsy qu'en ung spectacle publique, jamais rien ne se trouve parfait si tout ce qui appartient au theatre n'est ainsy fait et dispose comme la raison le veult, dont advient que ce qui est propose au dict theatre, semble beaucoup plus excellent et naturel, quand les spectateurs peuvent veoir tous et egalement, et sans fascherie qu'ilz puissent recepvoir du vent, pluye, ou soleil qu'il face : au moyen desquelles chose pourroyent quelquesfois les administrateurs estre retardez de leur operation. ${ }^{66}$

Il continue par la construction imaginaire d'un amphithéâtre de dissection, composé selon les préceptes de Vitruve, Alberti et Palladio, qui suivent les "vestiges " des Anciens. Au-delà de la commodité de la vue, dans une épistémologie de l'autopsie, la théâtralité du discours scientifique engage une réflexion sur l'espace qui donne sens au texte, et qui transforme le mot en parole. Dès la préface, Charles Estienne défend l'usage des illustrations (magnifiques gravures en pleine page de son traité in folio) par la valeur de la représentation, qui donne parole et, surtout, parole présente :

Car si les escriptz contentent l'esprit et la memoire, aussy pouvons nous dire que la peinture contentera l'oeil et la veue de la chose absente, aultant ou a peu pres comme si elle estoit presente. Les escriptz supplient la parolle : et les protraicts (combien que muets) portent la forme et facon des choses devant les yeulx, en sorte qu'ilz nont aultre mestier de parolle. ${ }^{67}$

Théâtre du savoir grâce aux images qui sont intégrées à sa structure, le livre de La Dissection se situe au-delà de «l'esprit et de la mémoire », que transcrit l'écrit : la parole vivante est celle qui est donnée à voir, « devant les yeux » sur

65. Charles Estienne, La Dissection des parties du corps humain (Paris : Simon de Colines, 1546), traduction du traité latin De Dissection Partium Humani Corporis (Paris : Simon de Colines, 1545).

66. Charles Estienne, La Dissection (1546), p. 373.

67. Charles Estienne, La Dissection (1546), p. 1. 
la scène des illustrations. Ainsi, texte et image semblent se répondre comme l'interprétation (écrite) et la traduction (jouée) : ils constituent, dans le jeu de leur complémentarité, la représentation complète du sens. Pareille poétique de l'expérience de la parole se retrouve dans l'Abbrégé de l'histoire des vicontes et ducz de Milan, recueil historique tiré de Paul Jove publié par Charles Estienne en 1552 et accompagné de gravures. Dès l'épître dédicatoire, l'auteur évoque la « vive voix » de la lecture et la compare à l'image :

Mais je croy Monseigneur, puisque la vive voix ou lecture de telle description, donne si grand contentement aux bons espritz, qu'encore plus le plaisir leur en doibt acroistre, quand l'histoire est accompaignee des pourtraicts et figures, prinses apres le naturel de ceulx, desquels les faicts sont en icelle recitez. ${ }^{68}$

La vérité du portrait «pris après le naturel » est pareille à celle de la parole prononcée. Une fois encore, le texte écrit est présenté comme une trace, lacunaire, sans vie, qui demande la parole et la scène des images pour prendre vie.

Dès lors, le long et laborieux travail de l'édition annotée, souvent invisible, destiné à rester muet, est celui de l'écrit, de la leçon, de la coulisse. Sur scène, résonne la parole qui donne vie à la lettre morte du texte. La représentation théâtrale, ainsi pensée, réalise par l'énonciation (les conditions de production de la parole) une appropriation du texte par les nouveaux locuteurs : elle constitue le modèle humaniste du renouveau de la parole des Anciens.

L'Andrienne est une comédie de filiation (on découvre qui est l'enfant de qui), de reconnaissance (on déclare de quel enfant on est le père) et de mariage (on célèbre l'union des parents, enfants, amants et amis enfin tous reconnus sur scène). Dans ce jeu de masques et de malentendus, que les humanistes aimaient à faire représenter par leur élèves et enfants, se raconte sur scène la reconnaissance d'une culture nouvelle dans une ancienne culture nouvelle, qui n'est autre que la reconnaissance de soi dans la parole de l'autre : sur cette scène humaniste de « retour aux Anciens », c'est la parole qui donne vie et sens. Jouer, c'est devenir... Magie du théâtre qui fait chatoyer savoirs et identités, la nouvelle comédie nouvelle de Charles Estienne représenterait bien, peut-être, 
la renaissance des lettres anciennes devenues paroles modernes. L'intérêt de Charles Estienne pour le théâtre est alors, également, un intérêt pour la traduction du théâtre, où la médiation devient visible hors de la représentation, dans les coulisses du livret explicatif.

Homme de lettres, de savoir, et d'édition, Charles Estienne donne par sa pratique de la traduction une poétique du partage de la parole, où se rencontrent pédagogie et plaisir, leçon et littérature : humaniste, il fait œuvre - discrète de passeur, d'éditeur, d'interprète. Dans la paraphrase pour la scène, il rend visible ces médiations, autrement oubliées, de la renaissance des lettres. Bien au-delà des répétitions chez les précepteurs, l'Andrienne donne naissance à un espace humaniste : sur cette scène, la lecture devient parole, le livre, théâtre et le moderne, ancien.

Quant au bon Terence, la mignardise, et les graces du langage Latin, je le trouve admirable à representer au vif les mouvemens de l'ame, et la condition de nos mœurs : à toute heure nos actions me rejettent à luy : Je ne le puis lire si souvent que je n'y trouve quelque beauté et grace nouvelle. ${ }^{69}$

69. Montaigne, Essais, 2, 10 (http://artflsrv02.uchicago.edu/cgi-bin/philologic/getobject.pl?c.0:3:9. montaigne consulté le $1^{\text {er }}$ novembre 2016). 
